Singularity theorems from weakened energy conditions

This article has been downloaded from IOPscience. Please scroll down to see the full text article.

2011 Class. Quantum Grav. 28125009

(http://iopscience.iop.org/0264-9381/28/12/125009)

View the table of contents for this issue, or go to the journal homepage for more

Download details:

IP Address: 72.144.81.219

The article was downloaded on 05/05/2011 at 10:35

Please note that terms and conditions apply. 


\title{
Singularity theorems from weakened energy conditions
}

\author{
Christopher J Fewster $^{1}$ and Gregory J Galloway ${ }^{2}$ \\ ${ }^{1}$ Department of Mathematics, University of York, Heslington, York YO10 5DD, UK \\ 2 Department of Mathematics, University of Miami, Coral Gables, FL, USA \\ E-mail: chris.fewster@york.ac.uk and galloway@math.miami.edu
}

Received 10 January 2011, in final form 30 March 2011

Published 4 May 2011

Online at stacks.iop.org/CQG/28/125009

\begin{abstract}
We establish analogues of the Hawking and Penrose singularity theorems based on (a) averaged energy conditions with exponential damping; (b) conditions on local stress-energy averages inspired by the quantum energy inequalities satisfied by a number of quantum field theories. As particular applications, we establish singularity theorems for the Einstein equations coupled to a classical scalar field, which violates the strong energy condition, and the nonminimally coupled scalar field, which also violates the null energy condition.
\end{abstract}

PACS numbers: $04.20 . \mathrm{Dw}, 04.40 . \mathrm{Nr}$

\section{Introduction}

The singularity theorems proved by Penrose and Hawking in the mid-1960s [1-3] represented a major advance over previous work, which was restricted to situations of high symmetry and particular matter models. At the technical level, this advance was achieved by significant development of the geometrical apparatus; at the physical level, however, the key idea was to replace particular matter models by generic energy conditions, which drive geodesic congruences to focal points. While many classical matter models obey the classical energy conditions, such as perfect fluids and the electromagnetic field, there are exceptions. For example, the Klein-Gordon field with nonzero mass fails to satisfy the strong energy condition (SEC; the hypothesis for the Hawking and Hawking-Penrose theorems if the cosmological constant vanishes), while the nonminimally coupled Klein-Gordon field also violates the null energy condition (NEC; the hypothesis of the Penrose theorem). See [4] for a critical assessment of the status of classical energy conditions and [5] for a review of singularity theorems and commentary on energy conditions in that context.

One of the main limitations of the energy conditions is that they are incompatible with quantum field theory, as has long been known [6]. Examples of states with locally negative expectation values for the energy density are easily constructed [7] and in a large class of 
quantum field theories the energy density at any given point is unbounded from below as the quantum state varies (see [8] and references therein). Moreover, recent results in twodimensional conformal field theory show that individual measurements of weighted spacetime averages of energy density in the vacuum state yield negative values with probabilities approaching $90 \%$ in some cases [9].

For these reasons a number of authors have investigated singularity theorems under weakened energy conditions. Examples include [10-13], in which various averaged energy conditions are considered, particularly the averaged null energy condition (ANEC), i.e.

$$
\int_{\gamma} T_{a b} \gamma^{\prime a} \gamma^{\prime b} \mathrm{~d} \lambda \geqslant 0
$$

where $\gamma$ is a complete (or sometimes future- or past-complete) null geodesic in an affine parameterization. (Via the Einstein equations, these conditions translate immediately to conditions on the Ricci tensor.) The definition of the integral requires some care; it is usually interpreted as a lim inf of the integrals over finite regions (as in [12]) or regularized by inserting a mollifying factor $f\left(\lambda / \lambda_{0}\right)^{2}$ in the integrand (for $f$ in a suitable function class) and taking the lim inf as $\lambda_{0} \rightarrow \infty$ [13]. On the other hand, a different approach (see 7.21-22 of [14] and [15]) is to suppose a lower bound on $R_{a b} t^{a} t^{b}$ for all unit timelike vectors and sufficient initial contraction for focussing to occur.

In this paper, we will establish singularity theorems under conditions that combine ideas from these two approaches but also extend them to allow for situations in which the integrand becomes unboundedly negative. Roughly speaking, we replace the ANEC integral by the requirement that for a future-complete null geodesic $\gamma:[0, \infty) \rightarrow M$,

$$
\int_{\gamma} \mathrm{e}^{-c \lambda} T_{a b} \gamma^{\prime a} \gamma^{\prime b} \mathrm{~d} \lambda-\frac{c}{2}
$$

should be finite for some $c>0$ (with the integral understood as above) and dominate the initial expansion of suitable future-complete null congruences in spacetime $M$. This leads to a generalization of the Penrose theorem, which is stated precisely as theorem 5.2; a similar generalization of Hawking's theorem is given as theorem 5.1. In section 6, we apply these results to prove a Hawking singularity theorem for the massive minimally coupled KleinGordon field (even though it can violate the SEC), at least provided that the field amplitude is bounded or, more generally, of exponential type. Under similar conditions on the amplitude of the field and its derivatives, we also prove a Penrose singularity theorem for the nonminimally coupled Klein-Gordon field (even though it can violate the NEC). Our results are based on criteria, derived in section 2, for the nonexistence of global solutions to Riccati equations that generalize arguments of [16] by inclusion of the exponential as an integrating factor (one could equally consider other functions in this context). This idea is used to generalize arguments of [13] in section 3 and the relevant singularity theorems are proved in section 5.

In section 4, we consider how exponentially damped energy conditions of the type required can be derived from bounds on local energy averages similar to those satisfied by quantum fields, known as quantum energy inequalities (QEIs) or quantum inequalities (QIs). Inequalities of this type, which express limitations on the magnitude and duration of violations of the classical energy conditions, were first mooted by Ford [17] and have since been established in a number of quantum field theory models (see $[8,18]$ for reviews). In four-dimensional Minkowski space, for example, the energy density of a free real scalar field obeys the bound

$$
\int_{-\infty}^{\infty}\left\langle T_{a b} \gamma^{\prime a} \gamma^{\prime b}\right\rangle_{\psi} f(t)^{2} \mathrm{~d} t \geqslant-\frac{1}{16 \pi^{2}} \int_{-\infty}^{\infty} f^{\prime \prime}(t)^{2} \mathrm{~d} t
$$


for any smooth compactly supported real-valued function $f$ and any Hadamard state $\psi[19,20]^{3}$. Here, $\gamma$ is any complete timelike geodesic in a proper time parameterization. Under rescaling of $f$, the bound scales as the inverse fourth power of the sampling timescale $\tau$; this is consistent with the unboundedness below of the energy density at points (in the limit $\tau \rightarrow 0$ ) and also gives an averaged weak energy condition in the limit $\tau \rightarrow \infty$. Similar results are known for other free fields.

Although analogous results hold in curved spacetimes, the bounds are more complicated in form. For example, the bound given in [22] involves local Fourier transforms of distributions formed from the first few terms in the expansion of the Hadamard parametrix, and is valid for averaging within suitable domains that typically have compact closure. However, it is expected on physical grounds [23] (and borne out in concrete examples) that averages over timescales smaller than local curvature length scales are constrained by bounds taking the same form as in Minkowski space. In the bounds of [22], for example, the first term in the Hadamard expansion will dominate the others on sufficiently small scales, and becomes well approximated by the expression in Minkowski space. This motivates the study of energy conditions in which local averages of the energy density or other stress-energy quantities are bounded from below in terms of $L^{2}$-norms of derivatives of the averaging function, perhaps with constraints on the support or after the subtraction of some reference function (as in the so-called difference QEIs). In section 4 we will derive (variants of) the exponentially damped energy conditions from local energy conditions of this type. This prepares the way for a more detailed study, which would have to quantify the scales over which the QEI bounds can be replaced by these simpler estimates. We also emphasize that, while our local conditions are motivated by QEIs, they do not coincide directly with them; see section 6 for discussion on this point.

\section{Criteria for nonexistence of global solutions to the Riccati equation}

We begin with a slight modification of an argument given in [16], lemma 3.

Lemma 2.1. Consider the initial value problem

$$
\begin{aligned}
& \dot{z}=\frac{z^{2}}{q}+p, \\
& z(0)=z_{0}
\end{aligned}
$$

where $q(t)$ and $p(t)$ are continuous on $[0, \infty)$, and $q(t)>0$ on $[0, \infty)$. If

$$
\int_{0}^{\infty} \frac{\mathrm{d} t}{q(t)}=+\infty \quad \text { and } \quad \liminf _{T \rightarrow+\infty} \int_{0}^{T} p(t) \mathrm{d} t>-z_{0},
$$

then (2.1) has no solution on $[0, \infty)$.

Proof. Suppose there is a solution $z(t)$ on $[0, \infty)$. By hypothesis, there exists $t_{1} \geqslant 0$ such that

$$
\int_{0}^{t} p\left(t^{\prime}\right) \mathrm{d} t^{\prime}>-z_{0}
$$

for all $t \in\left[t_{1}, \infty\right)$. Integrating the differential equation,

$$
z(t)=\int_{0}^{t} \frac{z\left(t^{\prime}\right)^{2}}{q\left(t^{\prime}\right)} \mathrm{d} t^{\prime}+\int_{0}^{t} p\left(t^{\prime}\right) \mathrm{d} t^{\prime}+z_{0}>\int_{0}^{t} \frac{z\left(t^{\prime}\right)^{2}}{q\left(t^{\prime}\right)} \mathrm{d} t^{\prime}
$$

3 Units with $\hbar=c=G=1$ are used throughout. Our geometrical conventions are those of [21]. 
for $t \geqslant t_{1}$. Introducing $R(t)=\int_{0}^{t} z\left(t^{\prime}\right)^{2} / q\left(t^{\prime}\right) \mathrm{d} t^{\prime}$, we see that $R$ is nonnegative and obeys the differential inequality

$$
\dot{R}=\frac{z^{2}}{q}>\frac{R^{2}}{q}
$$

for $t \geqslant t_{1}$. Accordingly, we have $R(t)>0$ for all $t>t_{1}$. Fixing any $t_{2}>t_{1}$, we now have

$$
\frac{1}{R\left(t_{2}\right)} \geqslant \frac{1}{R\left(t_{2}\right)}-\frac{1}{R(t)}=\int_{t_{2}}^{t} \frac{\dot{R}}{R^{2}} \mathrm{~d} t>\int_{t_{2}}^{t} \frac{\mathrm{d} t}{q}
$$

for $t>t_{2}$. However, the right-hand side is unbounded as $t \rightarrow \infty$ and we obtain a contradiction.

Here, 'no solution' means that we have $z(t) \rightarrow+\infty$ as $t \rightarrow t_{*}^{-}<\infty$. Note that

$$
z(t) \geqslant z_{0}+\int_{0}^{t} p\left(t^{\prime}\right) \mathrm{d} t^{\prime}
$$

for all $t$ for which the solution exists, so $z(t) \rightarrow-\infty$ cannot occur at finite time. (It is easily shown that divergence is the only way in which the solution can break down at finite times.)

As a digression from our main development, we note the following consequence.

Corollary 2.2. If, in lemma 2.1, the integral condition on $p$ is replaced by

$$
z_{0}+\inf _{T \geqslant 0} \int_{0}^{T} p(t) \mathrm{d} t=\alpha>0
$$

then (2.1) has no solution on $[0, \tau]$, where $\tau$ is the unique solution to

$$
\int_{0}^{\tau} \frac{\mathrm{d} t^{\prime}}{q\left(t^{\prime}\right)}=\frac{2}{\alpha}
$$

In particular, this applies if the negative part $p_{-}(t)=\min \{0, p(t)\}$ is integrable and $z_{0}+\int_{0}^{\infty} p_{-}(t) \mathrm{d} t=\alpha>0$.

Proof. Following the proof of the lemma, we may take $t_{1}=0$ and deduce from the hypothesis and equation (2.2) that $z(t) \geqslant \alpha$ for all $t \in[0, \infty)$ and hence that

$$
R\left(t_{2}\right) \geqslant \alpha^{2} \int_{0}^{t_{2}} \frac{\mathrm{d} t^{\prime}}{q\left(t^{\prime}\right)}
$$

for any $t_{2}>0$. Inequality (2.3) now asserts that

$$
\frac{1}{\alpha^{2}}>\left(\int_{0}^{t_{2}} \frac{\mathrm{d} t^{\prime}}{q\left(t^{\prime}\right)}\right)\left(\int_{t_{2}}^{t} \frac{\mathrm{d} t^{\prime}}{q\left(t^{\prime}\right)}\right)
$$

for all $0<t_{2} \leqslant t$ and the intermediate value theorem allows us to find $t_{2}$ such that both factors on the right-hand side are equal to $\frac{1}{2} \int_{0}^{t} q\left(t^{\prime}\right)^{-1} \mathrm{~d} t^{\prime}$, establishing the required result. Finally, if $p_{-}$is integrable, it is clear that $\int_{0}^{T} p(t) \mathrm{d} t \geqslant \int_{0}^{\infty} p_{-}(t) \mathrm{d} t$ for all $T$.

Remark. In the standard singularity theorems, $q(t) \equiv n-1$ for timelike geodesic congruences and $q(t) \equiv n-2$ for null geodesic congruences, where $n$ is the spacetime dimension. The upper bound on the parameter time until blow-up is therefore $\tau=2(n-1) / \alpha$ or $2(n-2) / \alpha$ respectively. Tighter bounds can be obtained in this case, by a more involved argument [24].

We now weaken the conditions on $p$; for simplicity we also fix $q$ to be a constant. 
Lemma 2.3. Consider the initial value problem

$$
\begin{aligned}
& \dot{z}=\frac{z^{2}}{s}+r, \\
& z(0)=z_{0}
\end{aligned}
$$

where $r(t)$ is continuous on $[0, \infty)$, and $s>0$ is constant. If there exists $c \geqslant 0$ such that $s$

$$
z_{0}-\frac{c}{2}+\liminf _{T \rightarrow+\infty} \int_{0}^{T} \mathrm{e}^{-2 c t / s} r(t) \mathrm{d} t>0
$$

then (2.4) has no solution on $[0, \infty)$.

Proof. Again, assume the contrary. Then, $y(t)=(z(t)-c) \mathrm{e}^{-2 c t / s}$ solves

$$
\begin{aligned}
& \dot{y}=\frac{y^{2}}{s \mathrm{e}^{-2 c t / s}}+\mathrm{e}^{-2 c t / s}\left(r(t)+c^{2} / s\right) \\
& y(0)=z_{0}-c
\end{aligned}
$$

on $[0, \infty)$. This equation is of the form $(2.1)$, with $q(t)=s \mathrm{e}^{-2 c t / s}$ and $p(t)=$ $\mathrm{e}^{-2 c t / s}\left(r(t)+c^{2} / s\right)$. Clearly, $\int_{0}^{\infty} \mathrm{d} t / q(t)=\infty$; as we also have

$$
\begin{aligned}
\liminf _{T \rightarrow+\infty} \int_{0}^{T} & \mathrm{e}^{-2 c t / s}\left(r(t)+c^{2} / s\right) \mathrm{d} t \geqslant \liminf _{T \rightarrow+\infty} \int_{0}^{T} \mathrm{e}^{-2 c t / s} r(t) \mathrm{d} t+\liminf _{T \rightarrow+\infty} \int_{0}^{T} \mathrm{e}^{-2 c t / s} c^{2} / s \mathrm{~d} t \\
& =\frac{c}{2}+\liminf _{T \rightarrow+\infty} \int_{0}^{T} \mathrm{e}^{-2 c t / s} r(t) \mathrm{d} t \\
& >c-z_{0}=-y(0)
\end{aligned}
$$

by the hypothesis, lemma 2.1 entails that $(2.5)$ has no solution on $[0, \infty)$ and we have obtained a contradiction.

For example, suppose that $r(t) \geqslant-A \mathrm{e}^{B t}$ for $A, B>0$. Then, for $c>B s / 2$ we have

$$
-\frac{c}{2}+\liminf _{T \rightarrow+\infty} \int_{0}^{T} \mathrm{e}^{-2 c t / s} r(t) \mathrm{d} t \geqslant-\frac{c}{2}-\frac{A s}{2 c-B s},
$$

the right-hand side of which has a maximum of $-(\sqrt{A s}+B s / 4)$ at $c=\sqrt{A s}+B s / 2$. Accordingly, (2.4) has no solution on $[0, \infty)$ if

$$
z_{0}>\sqrt{A s}+\frac{B s}{4}
$$

As an explicit example, consider $r(t)=-\mathrm{e}^{2 t}, s=1$ (i.e. $A=1, B=2$ ). The above inequality establishes nonexistence of global solutions for $z_{0}>3 / 2$; in fact, the equation can be solved in terms of modified Bessel functions as

$$
z(t)=\frac{\mathrm{d}}{\mathrm{d} t} \log \left(\left[I_{0}\left(e^{t}\right) K_{1}(1)+K_{0}\left(e^{t}\right) I_{1}(1)\right]-\left[I_{0}\left(e^{t}\right) K_{0}(1)-K_{0}\left(e^{t}\right) I_{0}(1)\right] z_{0}\right)
$$

and it is easy to see that $z(t)$ has a singularity at finite time if and only if

$$
z_{0}>\inf _{t \geqslant 0} \frac{I_{0}\left(e^{t}\right) K_{1}(1)+K_{0}\left(e^{t}\right) I_{1}(1)}{I_{0}\left(e^{t}\right) K_{0}(1)-K_{0}\left(e^{t}\right) I_{0}(1)}=\frac{K_{1}(1)}{K_{0}(1)}=1.429 \ldots,
$$

which is consistent with the estimate provided by lemma 2.3 .

We also have the following consequence.

Corollary 2.4. If, in lemma 2.3, the integral condition on $r$ is replaced by

$$
z_{0}-c+\inf _{T \geqslant 0} \int_{0}^{T} \mathrm{e}^{-2 c t / s}\left(r(t)+c^{2} / s\right) \mathrm{d} t=\alpha>0
$$


then (2.4) has no solution on $[0, \tau]$, where

$$
\tau=\frac{s}{2 c} \log (1+4 c / \alpha)
$$

In particular, this applies if the negative part $r_{-}(t)=\min \{0, r(t)\}$ is such that $r_{-}(t) \mathrm{e}^{-2 c t / s}$ is integrable and $z_{0}-c+\int_{0}^{\infty} \mathrm{e}^{-2 c t / s} r_{-}(t) \mathrm{d} t=\alpha>0$.

Proof. Following the proof of lemma 2.3, the hypothesis implies that

$$
y(0)+\inf _{T \geqslant 0} \int_{0}^{T} p(t) \mathrm{d} t=\alpha>0
$$

and we may apply corollary 2.2 to find that there is no solution to equation $(2.4)$ on $[0, \tau]$, where $\tau$ solves

$$
\frac{2}{\alpha}=\int_{0}^{\tau} \frac{\mathrm{d} t^{\prime}}{q\left(t^{\prime}\right)}=\frac{\mathrm{e}^{2 c \tau / s}-1}{2 c},
$$

proving the first statement. The second statement follows from the elementary bound $\int_{0}^{T} \mathrm{e}^{-2 c t / s}\left(r(t)+c^{2} / s\right) \mathrm{d} t \geqslant \int_{0}^{\infty} \mathrm{e}^{-2 c t / s} r_{-}(t) \mathrm{d} t$ for all $T \in[0, \infty)$.

For cases in which $\alpha \gg c$, the bound is approximately $2 s / \alpha$, which is the same as in the remark following corollary 2.2 .

\section{A generalization of the Wald-Yurtsever argument}

Wald and Yurtsever [13] consider hypotheses on weighted averages of the forcing term in the Riccati equation (see the lemma in that reference). Here, we generalize this further, after the pattern of lemma 2.3. Fix a real-valued compactly supported function $g \in C_{0}^{\infty}(\mathbb{R})$ with $g(t)=1$ on $[0,1]$, and $g$ nonincreasing on $\mathbb{R}^{+}$.

Lemma 3.1. Suppose that a solution to (2.4) exists on (all of) $t \in[0, \infty)$. If $r(t)$ is nonconstant, then

$$
\limsup _{\tau \rightarrow \infty} \int_{0}^{\infty} \mathrm{e}^{-2 c t / s} r(t) g(t / \tau)^{2} \mathrm{~d} t<\frac{c}{2}-z_{0}
$$

for all $c \geqslant 0$. If $r(t) \equiv r$ is constant, the same conclusion holds provided $c \neq z_{0}$ or $c^{2} / s+r \neq 0$.

\section{Remarks.}

(1) As the lim inf of any function is always less than its lim sup we may rather trivially replace lim sup by lim inf in the statement of this result.

(2) The $c=0$ case essentially corresponds to the result in [13].

Proof. If a solution to (2.4) exists for all $t$, then we know that (2.5) has a solution on $[0, \infty)$. Rearranging, and integrating against $g(t / \tau)^{2}$,

$$
\begin{aligned}
& I_{c}(\tau):=\int_{0}^{\infty} \mathrm{e}^{-2 c t / s} r(t) g(t / \tau)^{2} \mathrm{~d} t \\
& \quad=\int_{0}^{\infty} \dot{y}(t) g(t / \tau)^{2} \mathrm{~d} t-\frac{c^{2}}{s} \int_{0}^{\infty} \mathrm{e}^{-2 c t / s} g(t / \tau)^{2} \mathrm{~d} t-R_{c}(\tau),
\end{aligned}
$$

where, as before, $y(t)=(z(t)-c) \mathrm{e}^{-2 c t / s}$ obeys equation (2.5) and

$$
R_{c}(\tau)=\frac{1}{s} \int_{0}^{\infty} y(t)^{2} \mathrm{e}^{2 c t / s} g(t / \tau)^{2} \mathrm{~d} t
$$

6 
increases (not necessarily strictly) as $\tau$ increases, and is strictly positive for all $\tau>0$ unless $y \equiv 0$, which can happen only if $c=z_{0}$ and $r(t) \equiv-c^{2} / s$. Integrating (3.1) by parts,

$I_{c}(\tau)=-y(0)-\frac{2}{\tau} \int_{0}^{\infty} y(t) g(t / \tau) \dot{g}(t / \tau) \mathrm{d} t-\frac{c^{2}}{s} \int_{0}^{\infty} \mathrm{e}^{-2 c t / s} g(t / \tau)^{2} \mathrm{~d} t-R_{c}(\tau)$.

As $y$ is continuous, both $\mathrm{e}^{c t / s} y(t) g(t / \tau)$ and $\mathrm{e}^{-c t / s} \dot{g}(t / \tau)$ are square integrable, and the Cauchy-Schwarz inequality gives the estimate

$$
\begin{gathered}
\left|\int_{0}^{\infty} y(t) g(t / \tau) \dot{g}(t / \tau) \mathrm{d} t\right| \leqslant\left(\frac{1}{s} \int_{0}^{\infty} y^{2} \mathrm{e}^{2 c t / s} g(t / \tau)^{2} \mathrm{~d} t\right)^{1 / 2}\left(s \int_{0}^{\infty} \mathrm{e}^{-2 c t / s} \dot{g}(t / \tau)^{2} \mathrm{~d} t\right)^{1 / 2} \\
\leqslant C \sqrt{\tau R_{c}(\tau)} \mathrm{e}^{-c \tau / s}
\end{gathered}
$$

where $C>0$ is defined by

$$
C^{2}=s \int_{0}^{\infty} \dot{g}(t)^{2} \mathrm{~d} t
$$

and we have used the fact that $\dot{g}(t / \tau)=0$ in $[0, \tau]$. We may also estimate

$$
\frac{c}{2}\left(1-\mathrm{e}^{-2 c \tau / s}\right) \leqslant \frac{c^{2}}{s} \int_{0}^{\infty} \mathrm{e}^{-2 c t / s} g(t / \tau)^{2} \mathrm{~d} t \leqslant \frac{c}{2} .
$$

Putting these estimates in (3.2), we now have

$$
I_{c}(\tau) \leqslant-y(0)-\frac{c}{2}\left(1-\mathrm{e}^{-2 c \tau / s}\right)-R_{c}(\tau)+2 C \mathrm{e}^{-c \tau / s} \sqrt{\frac{R_{c}(\tau)}{\tau}}
$$

and, estimating

$$
2 C \mathrm{e}^{-c \tau / s} \sqrt{\frac{R_{c}(\tau)}{\tau}} \leqslant \frac{R_{c}(\tau)}{2}+2 C^{2} \frac{\mathrm{e}^{-2 c \tau / s}}{\tau}
$$

by the AM-GM inequality, we arrive at

$$
I_{c}(\tau) \leqslant-y(0)-\frac{c}{2}\left(1-\mathrm{e}^{-2 c \tau / s}\right)-\frac{R_{c}(\tau)}{2}+2 C^{2} \frac{\mathrm{e}^{-2 c \tau / s}}{\tau} .
$$

If either (a) $r(t)$ is nonconstant, or (b) $r(t) \equiv r$ but $z_{0} \neq c$ or $c^{2} / s+r \neq 0$, then $y$ cannot be identically zero. It follows that $R_{c}(\tau)$ is strictly positive and increasing on $(0, \infty)$. Thus, there exists $\tau_{0}>0$ such that

$$
I_{c}(\tau)<-y(0)-\frac{c}{2}=\frac{c}{2}-z_{0}
$$

for all $\tau>\tau_{0}$, which proves the required result.

\section{QEI-inspired hypotheses}

Now let us consider a QEI-inspired condition: we suppose that the forcing function $r(t)$ is constrained by inequalities of the form

$$
\int_{-\infty}^{\infty}\left(r(t)-r_{0}(t)\right) f(t)^{2} \mathrm{~d} t \geqslant-\|\mid f\|^{2}
$$

for all real-valued $f \in C_{0}^{\infty}(\mathbb{R})$, where $r_{0}(t)$ is a fixed continuous real-valued function and $\||\cdot|\|$ is any Sobolev (semi-)norm of the form

$$
\|\| f\left\|^{2}=\sum_{\ell=0}^{L} Q_{\ell}\right\| f^{(\ell)} \|^{2}
$$


where $Q_{\ell} \in[0, \infty)$ are constants of the appropriate dimensions, $L \in \mathbb{N}$ and $\|\cdot\|$ is the $L^{2}$-norm.

In the analogy with QEIs, $r_{0}$ would be obtained from a reference state. As already mentioned, this constraint is inspired by, rather than exactly coinciding with, the known QEI results. See section 6 for more discussion.

Let $g$ be as above and $c \geqslant 0$. Choose a smooth real-valued function $h \in C^{\infty}(\mathbb{R})$, such that $\operatorname{supp} h \subset\left[-\tau_{0}, \infty\right)$ and $h(t)=\mathrm{e}^{-c t / s}$ on $[0, \infty)$. Then, for each $\tau>0$,

$$
f_{\tau}(t)= \begin{cases}\mathrm{e}^{-\mathrm{ct} / \mathrm{s}} \mathrm{g}(\mathrm{t} / \tau) & t>0 \\ h(t) & t<0\end{cases}
$$

defines a test-function $f_{\tau} \in C_{0}^{\infty}(\mathbb{R})$. If $c>0$, then the dominated convergence theorem entails that $\left\|\left|f_{\tau}\|\| \rightarrow\|\| h \|\right|\right.$ as $\tau \rightarrow \infty$ and we have

$\liminf _{\tau \rightarrow+\infty} I_{c}(\tau) \geqslant-\int_{-\tau_{0}}^{0} h(t)^{2}\left(r(t)-r_{0}(t)\right) \mathrm{d} t-\||| h \mid\|+\liminf _{\tau \rightarrow+\infty} \int_{0}^{\infty} \mathrm{e}^{-2 c t / s} r_{0}(t) g(t / \tau)^{2} \mathrm{~d} t$.

The same holds if $c=0$, provided that the coefficient $Q_{0}$ vanishes in the definition of \|\|$\cdot\|\|$. Note that ||$h|| \mid$ can be written as

$$
\left\||h \||=\sum_{\ell=0}^{L} Q_{\ell}\left(\frac{1}{2}(c / s)^{2 \ell-1}+\int_{-\tau_{0}}^{0}\left|h^{(\ell)}(t)\right|^{2} \mathrm{~d} t\right) .\right.
$$

The following result is now immediate.

Theorem 4.1. Suppose $r(t)$ obeys (4.1) and is nonconstant. Suppose there exist $c>0$, $\tau_{0}>0$ and $h \in C^{\infty}(\mathbb{R})$ obeying supp $h \subset\left[-\tau_{0}, \infty\right)$ and $h(t)=\mathrm{e}^{-c t / s}$ on $[0, \infty)$, for which $z_{0}-\frac{c}{2}+\liminf _{\tau \rightarrow+\infty} \int_{0}^{\infty} \mathrm{e}^{-2 c t / s} r_{0}(t) g(t / \tau)^{2} \mathrm{~d} t \geqslant \int_{-\tau_{0}}^{0} h(t)^{2}\left(r(t)-r_{0}(t)\right) \mathrm{d} t+\|\| h \mid \|^{2}$.

Then, (2.4) has no solution on all of $[0, \infty)$. If $r(t) \equiv r$ obeys (4.1), the same conclusion holds provided $c \neq z_{0}$ or $c^{2} / s+r \neq 0$. If $Q_{0}=0$, the hypothesis on c can be relaxed to $c \geqslant 0$.

Proof. By lemma 3.1, if there is a solution to $(2.4)$ on $[0, \infty)$, then

$$
\begin{aligned}
0 & >z_{0}-\frac{c}{2}+\liminf _{\tau \rightarrow \infty} I_{c}(\tau) \\
& \geqslant z_{0}-\frac{c}{2}+\liminf _{\tau \rightarrow+\infty} \int_{0}^{\infty} \mathrm{e}^{-2 c t / s} r_{0}(t) g(t / \tau)^{2} \mathrm{~d} t-\int_{-\tau_{0}}^{0} h(t)^{2}\left(r(t)-r_{0}(t)\right) \mathrm{d} t-\||| h \mid\|^{2} \\
& \geqslant 0
\end{aligned}
$$

by hypothesis, which is a contradiction.

The above theorem tells us that a focal point will occur if there is sufficient initial contraction. Note that the dependence on the actual matter distribution $r(t)$ is purely through its values in $\left[-\tau_{0}, 0\right]$, i.e. before the contraction $z_{0}$ is measured. At first sight it is strange that positive values of $r(t)-r_{0}$ require a larger value of $z_{0}$ : the reason is simply that the averaged nature of the energy condition means that large positive energies in the present allow large negative values in the future. The magnitude of initial contraction required is otherwise determined by the reference $r_{0}$ and the function $h$.

In some QEIs, the support of the sampling function is constrained, for example, to be small relative to local curvature length scales. For simplicity, let us suppose that there is some $\tau_{0}$ such that (4.1) is valid for all $f$ with support in an interval of length at most $2 \tau_{0}$. (This would correspond to conditions in which curvatures remain bounded; if they do not, this 
might be regarded as an indication of singular behaviour, albeit not in the sense of geodesic incompleteness.) We also restrict to a particular seminorm which would be most relevant to QEIs in four dimensions.

To discuss averages over longer timescales we will use a partition of unity. To this end, we will say that $\psi \in C_{0}^{\infty}(\mathbb{R})$ is a bump function if it is nonnegative, supported in $[0,3 / 2]$ and obeys $\psi(3 / 4+x)=\psi(3 / 4-x), \psi(x)^{2}+\psi(1 / 2-x)^{2}=1$ for $x \in[0,3 / 4]$; in particular, this gives $\psi=1$ on $[1 / 2,1]$. Then, the functions $\psi(x-n)^{2}(n \in \mathbb{Z})$ form a partition of unity on $\mathbb{R}$. Note that this partition involves the squares of the underlying functions. The main work is to show that this gives usable bounds on the exponentially damped averages appearing in lemma 3.1.

Proposition 4.2. Let $r_{0}$ be a fixed continuous function and suppose that $r(t)$ is nonconstant and obeys

$$
\int_{-\infty}^{\infty}\left(r(t)-r_{0}(t)\right) f(t)^{2} \mathrm{~d} t \geqslant-Q\left\|f^{\prime \prime}\right\|^{2}
$$

for any $f \in C_{0}^{\infty}((0, \infty))$ supported in an interval of length at most $2 \tau_{0}>0$. Let $\psi$ be a bump function. Then, for any $c>0$ we have

$$
\begin{aligned}
\liminf _{\tau \rightarrow+\infty} \int_{0}^{\infty} \mathrm{e}^{-2 c t / s} r(t) g(t / \tau)^{2} \mathrm{~d} t \geqslant \liminf _{\tau \rightarrow+\infty} \int_{0}^{\infty} \mathrm{e}^{-2 c t / s} r_{0}(t) g(t / \tau)^{2} \mathrm{~d} t \\
\quad-\int_{-\tau_{0}}^{0}\left(r(t)-r_{0}(t)\right) h(t)^{2} \mathrm{~d} t-Q\left(\left\|h^{\prime \prime}\right\|^{2}+\frac{1}{2}\left(\frac{c}{s}\right)^{3}+\frac{\left\|\psi^{\prime \prime}\right\|^{2}}{\tau_{0}^{3}\left(1-\mathrm{e}^{-2 c \tau_{0} / s}\right)}\right) .
\end{aligned}
$$

for all real-valued $h \in C_{0}^{\infty}\left(\left(-\tau_{0}, \tau_{0} / 2\right)\right)$ with $h(t)=\mathrm{e}^{-c t / s} \psi\left(1 / 2-t / \tau_{0}\right)$ on $t \geqslant 0$. The last term may be replaced by

$$
-Q\left(\int_{-\tau_{0}}^{0}\left|h^{\prime \prime}(t)\right|^{2} \mathrm{~d} t+\left(\frac{c}{s}\right)^{3}+\frac{\left\|\psi^{\prime \prime}\right\|^{2}}{\tau_{0}^{3}}\left(\frac{1}{2}+\frac{1}{\left(1-\mathrm{e}^{-2 c \tau_{0} / s}\right)}\right)\right) .
$$

We defer the proof to the end of this section. The following consequence is immediate, by the same reasoning as in theorem 4.1.

Theorem 4.3. Let $r_{0}$ be a fixed continuous function and $\psi$ be a bump function. Suppose $r(t)$ is nonconstant and obeys (4.2) for any $f \in C_{0}^{\infty}((0, \infty))$ supported in an interval of length at most $2 \tau_{0}>0$. If, for some $c>0$ and h obeying the conditions of proposition 4.2, we have

$$
z_{0} \geqslant \frac{c}{2}-(\text { RHS of }(4.3))
$$

then (2.4) has no solution on all of $[0, \infty)$. If $r(t) \equiv r$ obeys (4.2), the same conclusion holds provided $c \neq z_{0}$ or $c^{2} / s+r \neq 0$.

Remark. It is possible to find bump functions $\psi$ with $\left\|\psi^{\prime \prime}\right\|^{2} \sim 331$. To see this, consider the ansatz

$$
\psi(x)= \begin{cases}\sin (\theta(x)) & x \leqslant 1 / 4 \\ \cos (\theta(1 / 2-x)) & 1 / 4<x \leqslant 3 / 4 \\ \psi(3 / 2-x) & x>3 / 4\end{cases}
$$

for $\theta \in C^{\infty}(\mathbb{R}), \theta \equiv 0$ on $\mathbb{R}^{-}, \theta \geqslant 0$ and $\theta(1 / 4)=\pi / 4$, and $\theta^{(2 k)}(1 / 4)=0$ for all $k \in \mathbb{N}$, which ensure that $\psi$ is smooth at $x=1 / 4$. After a calculation, one finds that

$$
\left\|\psi^{\prime \prime}\right\|^{2}=2 \int_{0}^{1 / 4}\left(\theta^{\prime}(x)^{4}+\theta^{\prime \prime}(x)^{2}\right) \mathrm{d} t
$$


which corresponds to the Euler-Lagrange equation

$$
\theta^{\prime \prime \prime \prime}(x)-6 \theta^{\prime}(x)^{2} \theta^{\prime \prime}(x)=0
$$

together with boundary conditions $\theta(0)=\theta^{\prime}(0)=0, \theta(1 / 4)=\pi / 4, \theta^{\prime \prime}(1 / 4)=0$. Numerical solution of this ODE in Maple 14 gives the value $\left\|\psi^{\prime \prime}\right\|^{2}=330.97$. This does not extend to give a smooth solution to our original problem as $\theta^{\prime \prime}(0+)=44.56$; however, by considering a mollified function $\theta_{\epsilon}(x)=\theta(x) f(x / \epsilon)$ with $f \in C^{\infty}(\mathbb{R}), f(x)=0$ on $(-\infty, 0], f(x) \equiv 1$ on $[1, \infty)$, one may show that $\left\|\psi_{\epsilon}^{\prime \prime}\right\| \rightarrow\left\|\psi^{\prime \prime}\right\|$ as $\epsilon \rightarrow 0^{+}$[one uses estimates $\theta(x)=O\left(x^{2}\right)$, $\theta^{\prime}(x)=O(x), \theta^{\prime \prime}(x)=O(1)$ near $\left.x=0\right]$.

Proof of proposition 4.2. The idea of the proof is to use a partition of unity to decompose the integral

$$
J_{c}(\tau)=\int_{0}^{\infty} \mathrm{e}^{-2 c t / s}\left(r(t)-r_{0}(t)\right) g(t / \tau)^{2} \mathrm{~d} t,
$$

thereby obtaining a sum of integrals, each of which can be bounded below using (4.2) (only finitely many terms appear at any fixed value of $\tau$ ). The sum of the resulting lower bounds must be controlled as $\tau \rightarrow \infty$.

We start from the identity

$$
\begin{gathered}
\left(\frac{\mathrm{d}^{2}}{\mathrm{~d} t^{2}} \mathrm{e}^{-c t / s} \varphi(t)\right)^{2}=\mathrm{e}^{-2 c t / s}\left(\frac{c}{s}\right)^{4}\left\{\varphi(t)^{2}-2 \frac{s}{c} \frac{\mathrm{d}}{\mathrm{d} t}\left(\varphi^{2}\right)+2\left(\frac{s}{c}\right)^{2} \varphi^{\prime}(t)^{2}+\left(\frac{s}{c}\right)^{2} \frac{\mathrm{d}^{2}}{\mathrm{~d} t^{2}}\left(\varphi^{2}\right)\right. \\
\left.-2\left(\frac{s}{c}\right)^{3} \frac{\mathrm{d}}{\mathrm{d} t}\left(\left(\varphi^{\prime}\right)^{2}\right)+\left(\frac{s}{c}\right)^{4} \varphi^{\prime \prime}(t)^{2}\right\}
\end{gathered}
$$

For real-valued $\varphi \in C_{0}^{\infty}((0, \infty))$, we may integrate by parts and discard a nonpositive term to find

$$
\begin{gathered}
\int_{0}^{\infty}\left(\frac{\mathrm{d}^{2}}{\mathrm{~d} t^{2}} \mathrm{e}^{-c t / s} \varphi(t)\right)^{2} \mathrm{~d} t=\left(\frac{c}{s}\right)^{4} \int_{0}^{\infty} \mathrm{e}^{-2 c t / s}\left(\varphi(t)^{2}-2\left(\frac{s}{c}\right)^{2} \varphi^{\prime}(t)^{2}+\left(\frac{s}{c}\right)^{4} \varphi^{\prime \prime}(t)^{2}\right) \mathrm{d} t \\
\leqslant \int_{0}^{\infty} \mathrm{e}^{-2 c t / s}\left(\left(\frac{c}{s}\right)^{4} \varphi(t)^{2}+\varphi^{\prime \prime}(t)^{2}\right) \mathrm{d} t
\end{gathered}
$$

If, additionally, the support of $\varphi$ is contained in an interval of length at most $2 \tau_{0}$, we may apply (4.2) with $f(t)=\mathrm{e}^{-c t / s} g(t / \tau) \varphi(t)$ and $g$ chosen as above, to give

$$
\begin{aligned}
& \int_{0}^{\infty} \mathrm{e}^{-2 c t / s}\left(r(t)-r_{0}(t)\right) g(t / \tau)^{2} \varphi(t)^{2} \mathrm{~d} t \\
& \quad \geqslant-Q \int_{0}^{\infty} \mathrm{e}^{-2 c t / s}\left(\frac{c^{4}}{s^{4}} g(t / \tau)^{2} \varphi(t)^{2}+\left(\frac{\mathrm{d}^{2}}{\mathrm{~d} t^{2}} g(t / \tau) \varphi(t)\right)^{2}\right) \mathrm{d} t,
\end{aligned}
$$

where we have estimated $\left\|f^{\prime \prime}\right\|^{2}$ using (4.7), with $\varphi$ replaced by $g(t / \tau) \varphi(t)$. This estimate will be used for each term appearing in the partition of unity, to which we now turn.

Let $\varphi(t)=\psi\left(t / \tau_{0}\right)$, where $\psi$ is the chosen bump function. Defining $\varphi_{n}(t)=\varphi\left(t-n t_{0}\right)$, the $\varphi_{n}^{2}$ form a partition of unity so that $\sum_{n=0}^{\infty} \varphi_{n}(t)^{2} \equiv 1$ on $t>\tau_{0} / 2$ and at most two $\varphi_{n}(t)$ are nonzero at each $t$; each $\varphi_{n}$ has a support diameter of $3 \tau_{0} / 2$. We may now decompose the integral $J_{c}(\tau)$ using this partition, estimating each term using (4.8). This gives

$$
\begin{aligned}
J_{c}(\tau)= & \int_{0}^{\tau_{0} / 2} \mathrm{e}^{-2 c t / s}\left(r(t)-r_{0}(t)\right) g(t / \tau)^{2}\left(1-\varphi(t)^{2}\right) \mathrm{d} t+\sum_{n=0}^{\infty} \int_{0}^{\infty} \mathrm{e}^{-2 c t / s}(r(t) \\
& \left.\quad-r_{0}(t)\right) g(t / \tau)^{2} \varphi_{n}(t)^{2} \mathrm{~d} t \\
\geqslant & \int_{0}^{\tau_{0} / 2} \mathrm{e}^{-2 c t / s}\left(r(t)-r_{0}(t)\right) g(t / \tau)^{2}\left(1-\varphi(t)^{2}\right) \mathrm{d} t-Q \sum_{n=0}^{\infty} S_{n}(\tau)
\end{aligned}
$$


where

$$
S_{n}(\tau)=\int_{0}^{\infty} \mathrm{e}^{-2 c t / s}\left(\left(\frac{c}{s}\right)^{4} g(t / \tau)^{2} \varphi_{n}(t)^{2}+\left(\frac{\mathrm{d}^{2}}{\mathrm{~d} t^{2}} g(t / \tau) \varphi_{n}(t)\right)^{2}\right) \mathrm{d} t .
$$

Our task is now to control the sum $\sum_{n=0}^{\infty} S_{n}(\tau)$ in the limit $\tau \rightarrow \infty$. Now, for $\tau>(n+3 / 2) \tau_{0}, g(t / \tau) \equiv 1$ on the support of $\varphi_{n}$, and we obtain

$$
\begin{aligned}
S_{n}(\tau)=\int_{0}^{\infty} & \mathrm{e}^{-2 c t / s}\left(\left(\frac{c}{s}\right)^{4} \varphi_{n}(t)^{2}+\varphi_{n}^{\prime \prime}(t)^{2}\right) \mathrm{d} t \\
& \leqslant\left(\frac{c}{s}\right)^{4} \int_{0}^{\infty} \mathrm{e}^{-2 c t / s} \varphi_{n}(t)^{2} \mathrm{~d} t+\mathrm{e}^{-2 c n \tau_{0} / s}\left\|\varphi^{\prime \prime}\right\|^{2},
\end{aligned}
$$

where the exponential factor in the second term arises on account of the support properties of $\varphi_{n}$ and we use $\left\|\varphi_{n}^{\prime \prime}\right\|=\left\|\varphi^{\prime \prime}\right\|$. Provided the limit and sum can be exchanged (which will be justified below), we then have

$$
\lim _{\tau \rightarrow \infty} \sum_{n=0}^{\infty} S_{n}(\tau) \leqslant \sum_{n=0}^{\infty}\left(\frac{c}{s}\right)^{4} \int_{0}^{\infty} \mathrm{e}^{-2 c t / s} \varphi_{n}(t)^{2} \mathrm{~d} t+\left\|\varphi^{\prime \prime}\right\|^{2} \sum_{n=0}^{\infty} \mathrm{e}^{-2 c n \tau_{0} / s} .
$$

The first sum can be evaluated using the partition of unity property and the second is elementary, so

$$
\begin{aligned}
\lim _{\tau \rightarrow \infty} \sum_{n=0}^{\infty} S_{n}(\tau) & \leqslant\left(\frac{c}{s}\right)^{4} \int_{0}^{\tau_{0} / 2} \mathrm{e}^{-2 c t / s}\left(\varphi(t)^{2}-1\right) \mathrm{d} t+\left(\frac{c}{s}\right)^{4} \int_{0}^{\infty} \mathrm{e}^{-2 c t / s} \mathrm{~d} t+\frac{\left\|\varphi^{\prime \prime}\right\|^{2}}{1-\mathrm{e}^{-2 c \tau_{0} / s}} \\
& \leqslant \frac{1}{2}\left(\frac{c}{s}\right)^{3}+\frac{\left\|\varphi^{\prime \prime}\right\|^{2}}{1-\mathrm{e}^{-2 c \tau_{0} / s}},
\end{aligned}
$$

where we discard the nonpositive contribution from $\varphi^{2}-1$ in the last step.

Assembling the results so far, we have

$\liminf _{\tau \rightarrow+\infty} J_{c}(\tau) \geqslant \int_{0}^{\tau_{0} / 2} \mathrm{e}^{-2 c t / s}\left(r(t)-r_{0}(t)\right)\left(1-\varphi(t)^{2}\right) \mathrm{d} t-Q\left(\frac{1}{2}\left(\frac{c}{s}\right)^{3}+\frac{\left\|\psi^{\prime \prime}\right\|^{2}}{\tau_{0}^{3}\left(1-\mathrm{e}^{-2 c \tau_{0} / s}\right)}\right)$.

Taking $h$ as specified in the hypotheses, we note that $h(t)^{2}=\mathrm{e}^{-2 c t / s}\left(1-\varphi(t)^{2}\right)$ on $t \geqslant 0$. Applying equation (4.2) with $h$ in place of $f$, we obtain

$\liminf _{\tau \rightarrow+\infty} J_{c}(\tau) \geqslant-\int_{-\tau_{0}}^{0}\left(r(t)-r_{0}(t)\right) h(t)^{2} \mathrm{~d} t-Q\left(\left\|h^{\prime \prime}\right\|^{2}+\frac{1}{2}\left(\frac{c}{s}\right)^{3}+\frac{\left\|\psi^{\prime \prime}\right\|^{2}}{\tau_{0}^{3}\left(1-\mathrm{e}^{-2 c \tau_{0} / s}\right)}\right)$.

Finally, because

$$
\liminf _{\tau \rightarrow+\infty} I_{c}(\tau) \geqslant \liminf _{\tau \rightarrow+\infty} J_{c}(\tau)+\liminf _{\tau \rightarrow+\infty} \int_{0}^{\infty} \mathrm{e}^{-2 c t / s} r_{0}(t) \mathrm{d} t
$$

we obtain the required result (4.3). The alternative form of the bound arises if we estimate $\left\|h^{\prime \prime}\right\|^{2}$ by using (4.6) applied to $\varphi(t)=\psi\left(1 / 2-t / \tau_{0}\right)$ and integrating by parts on $[0, \infty)$ to obtain (4.7) (the boundary terms at $t=0$ cancel). Using the properties of $\psi$ we find

$$
\left\|h^{\prime \prime}\right\|^{2} \leqslant \int_{-\tau_{0}}^{0}\left|h^{\prime \prime}(t)\right|^{2} \mathrm{~d} t+\frac{1}{2}\left(\frac{c}{s}\right)^{3}+\frac{1}{2 \tau_{0}^{3}}\left\|\psi^{\prime \prime}\right\|^{2}
$$

which yields the alternative bound.

It remains to justify the exchange of limit and sum used above, for which we use a dominated convergence argument. We split $S_{n}(\tau)$ into the sum of two terms. For the first, we note that

$$
\int_{0}^{\infty} \mathrm{e}^{-2 c t / s} g(t / \tau)^{2} \varphi_{n}(t)^{2} \mathrm{~d} t \leqslant \mathrm{e}^{-2 c n \tau_{0} / s}\|\varphi\|^{2}
$$


because supp $\varphi_{n} \subset\left[n \tau_{0}, \infty\right)$. The second is regarded as the square of an $L^{2}$ norm, which can be estimated using the Leibniz rule and triangle inequalities as

$$
\left[\int_{0}^{\infty} \mathrm{e}^{-2 c t / s}\left(\frac{\mathrm{d}^{2}}{\mathrm{~d} t^{2}} g(t / \tau) \varphi_{n}(t)\right)^{2} \mathrm{~d} t\right]^{1 / 2} \leqslant \frac{C_{n}}{\tau^{2}}+\frac{C_{n}^{\prime}}{\tau}+C_{n}^{\prime \prime},
$$

where $C_{n}, C_{n}^{\prime}$ and $C_{n}^{\prime \prime}$ are the $L^{2}$ norms of $\mathrm{e}^{-c t / s} g^{\prime \prime}(t / \tau) \varphi_{n}(t), 2 \mathrm{e}^{-c t / s} g^{\prime}(t / \tau) \varphi_{n}^{\prime}(t)$ and $\mathrm{e}^{-c t / s} g(t / \tau) \varphi_{n}^{\prime \prime}(t)$ respectively. Setting $C=\max \left\{1,4\left\|g^{\prime}\right\|_{\infty}^{2},\left\|g^{\prime \prime}\right\|_{\infty}^{2}\right\}$ and again using the support properties of $\varphi_{n}$, we have

$\int_{0}^{\infty} \mathrm{e}^{-2 c t / s}\left(\frac{\mathrm{d}^{2}}{\mathrm{~d} t^{2}} g(t / \tau) \varphi_{n}(t)\right)^{2} \mathrm{~d} t \leqslant C \tau_{0} \mathrm{e}^{-2 c n \tau_{0} / s}\left(\frac{\|\psi\|}{\tau^{2}}+\frac{\left\|\psi^{\prime}\right\|}{\tau \tau_{0}}+\frac{\left\|\psi^{\prime \prime}\right\|}{\tau_{0}^{2}}\right)^{2}$.

Accordingly, there exists $K>0$ such that $0 \leqslant S_{n}(\tau) \leqslant K \mathrm{e}^{-2 c n \tau_{0} / s}$ for all $n \in \mathbb{N}_{0}$ and $\tau>\tau_{0}$, where $K$ is independent of $\tau$ and $n$. As this upper bound is evidently summable, the dominated convergence theorem permits us to exchange the limit and sum as claimed.

\section{Singularity theorems}

The results from the previous sections can be used to obtain refinements of some of the classical singularity theorems of Hawking and Penrose [21] that allow global violations of the classical energy conditions.

We first consider the cosmological setting.

Theorem 5.1. Let $M$ be a globally hyperbolic spacetime of dimension $n \geqslant 2$, and let $S$ be a smooth compact spacelike Cauchy surface for M. Suppose along each future complete unit speed timelike geodesic $\gamma:[0, \infty) \rightarrow M$ issuing orthogonally from $S$, there exists $c \geqslant 0$ such that

$$
\liminf _{T \rightarrow \infty} \int_{0}^{T} \mathrm{e}^{-2 c t /(n-1)} r(t) \mathrm{d} t>\theta(p)+\frac{c}{2},
$$

where $r(t):=\operatorname{Ric}\left(\gamma^{\prime}(t), \gamma^{\prime}(t)\right)=R_{a b} \gamma^{\prime a} \gamma^{\prime b}(t)$ and $\theta(p)$ is the expansion (i.e. mean curvature) of $S$ at $p=\gamma(0)$. Then, $M$ is future timelike geodesically incomplete.

\section{Remarks.}

(1) As usual, if one assumes that the Einstein equations,

$$
R_{a b}-\frac{1}{2} R g_{a b}=8 \pi T_{a b}
$$

hold, then the Ricci curvature term can be directly related to the energy-momentum tensor $T_{a b}$, and, hence, one should view (5.1) as an energy condition on spacetime.

(2) Note that the energy condition (5.1) is satisfied provided along each such timelike geodesic $\gamma$, the condition

$$
\liminf _{T \rightarrow \infty} \int_{0}^{T} r(t) \mathrm{d} t>\theta(p)
$$

(corresponding to $c=0$ ) holds. This condition emphasizes the fact that if $S$ is mean contracting, i.e. if $\theta$ is negative on $S$, then (5.1) can hold even if $r(t)$ is everywhere negative.

(3) Specializing further, if the strong energy condition holds, i.e. if $\operatorname{Ric}(X, X)=R_{a b} X^{a} X^{b} \geqslant$ 0 for all timelike vectors $X$, and if $S$ is everywhere mean contracting, then (5.1) holds, and we essentially recover Hawking's cosmological singularity theorem; cf theorem 4 in [21], p 272. (For convenience we have stated theorem 5.1 as a future singularity result rather than a past singularity result.) 
Proof of theorem 5.1. We construct an $S$-ray $\gamma$, i.e. a future inextendible timelike geodesic $\gamma$ emanating from a point on $S$ that realizes the Lorentzian distance to $S$ from each of its points, as follows. Choose a sequence of points $q_{n}$ which extends arbitrarily far into the future of $S .{ }^{4}$ By the properties of Cauchy surfaces, there exists a timelike geodesic segment $\gamma_{n}$ from $p_{n} \in S$ to $q_{n}$ that realizes the Lorentzian distance from $S$ to $q_{n}$. Since $S$ is compact, by taking a subsequence if necessary, we may assume that the sequence $p_{n}$ converges to a point $p \in S$. Let $\gamma:[0, a) \rightarrow M, a \in(0, \infty]$, be the future inextendible unit speed timelike geodesic issuing orthogonally from $p \in S$. The maximality of the $\gamma_{n}$ 's guarantees that $\gamma$ is an $S$-ray (see, for example, the proof of the sublemma in [25]).

Let $\rho: J^{+}(S) \rightarrow \mathbb{R}$ be the Lorentzian distance function from $S$,

$$
\rho(x)=d(S, x)=\sup _{y \in S} d(y, x)
$$

By global hyperbolicity, $\rho$ is continuous on $J^{+}(S)$, and will be smooth up to the focal cut locus of $S$ (see [26]). Since $\gamma$ is an $S$-ray, there are no focal points, or focal cut points, to $S$ along $\gamma$, which, by the lower semi-continuity of the $S$-distance-to-cut locus function [26], ensures that $\rho$ is smooth on a neighbourhood $U$ of $\gamma$. On this neighbourhood $u:=-\nabla \rho$ is a smooth future directed, geodesic, hypersurface orthogonal unit timelike vector field such that $u=\gamma^{\prime}$ along $\gamma$. Consider the expansion scalar $\theta=\operatorname{div} u$. Along $\gamma, \theta=\theta(t), t \in[0, a)$, obeys Raychaudhuri's equation (for an irrotational timelike congruence) [21]

$$
\frac{\mathrm{d} \theta}{\mathrm{d} t}=-\operatorname{Ric}\left(\gamma^{\prime}, \gamma^{\prime}\right)-2 \sigma^{2}-\frac{1}{n-1} \theta^{2},
$$

where $\sigma$ is the shear scalar.

We now observe that $\gamma$ is necessarily future incomplete. Suppose that $\gamma$ is future complete (i.e. suppose $a=\infty)$. Setting $z=-\theta, r=\operatorname{Ric}\left(\gamma^{\prime}, \gamma^{\prime}\right)+2 \sigma^{2}, s=n-1$ and $z(0)=-\theta(p)$ in (2.4), we see that lemma 2.3, together with the energy condition (5.1), implies that (5.3), with $\theta(0)=\theta(p)$, has no solution on $[0, \infty)$, which is a contradiction.

\section{Remarks.}

(1) Although slightly more complicated to state, lemma 3.1 and theorems 4.1 and 4.3 provide alternative energy conditions that yield singularity theorems similar to theorem 5.1.

(2) The assumption of global hyperbolicity in theorem 5.1 can be relaxed. It is sufficient to assume that $M$ admits a smooth compact acausal spacelike hypersurface $S$. In this case one can construct an $S$-ray $\gamma$ contained in the future domain of dependence $D^{+}(S)$ (see especially [25], main lemma), and use the fact that $S$ is a Cauchy surface for the total domain of dependence $D(S)$.

(3) We see from the proof that it would be sufficient for (5.1) to hold on $S$-rays.

We now consider an extension of the Penrose singularity theorem [21], theorem 1, to the energy conditions being considered here. Let $\Sigma$ be a codimension-two compact acausal spacelike submanifold in a spacetime $M$ of dimension $n \geqslant 3$. Under suitable orientation assumptions, there exist two smooth nonvanishing independent null normal vector fields, $\ell_{+}$ and $\ell_{-}$, along $\Sigma$, corresponding to outgoing and ingoing light-rays emanating from $\Sigma$. The null expansion scalars $\theta_{+}$and $\theta_{-}$on $\Sigma$ are obtained by taking the divergence of $\ell_{+}$and $\ell_{-}$, respectively, along $\Sigma$, and measure the instantaneous divergence of the outgoing and ingoing light rays emanating from $\Sigma$. In a strong gravitational field both $\theta_{+}$and $\theta_{-}$can be negative, in which case $\Sigma$ is called a trapped surface. According to the Penrose singularity theorem, if $\Sigma$

4 More precisely, if $h$ is a complete background Riemannian metric on $M$, choose $q_{n}$ in $J^{+}(S)$ so that the $h$-distance from $S$ to $q_{n}$ tends to infinity as $n \rightarrow \infty$. 
is a trapped surface in a spacetime $M$ having a noncompact Cauchy surface and satisfying the null energy condition, then $M$ is future null geodesically incomplete.

The following generalizes the Penrose singularity theorem (see also [27], theorem 2).

Theorem 5.2. Let $M$ be a spacetime of dimension $n \geqslant 3$ with a noncompact Cauchy surface $S$. Let $\Sigma$ be a smooth compact acausal spacelike submanifold of $M$ of codimension 2, with null expansion scalars $\theta_{ \pm}$associated with the future directed null normal vector fields $\ell_{ \pm}$. Suppose along each future complete affinely parameterized null geodesic $\eta:[0, \infty) \rightarrow M$, issuing orthogonally from $\Sigma$ with initial tangent $\ell_{ \pm}$, there exists $c \geqslant 0$ such that

$$
\liminf _{T \rightarrow \infty} \int_{0}^{T} \mathrm{e}^{-2 c t /(n-2)} r(t) \mathrm{d} t>\theta_{ \pm}(p)+\frac{c}{2},
$$

where $p=\eta(0)$ and $r(t):=\operatorname{Ric}\left(\eta^{\prime}(t), \eta^{\prime}(t)\right)=R_{a b} \eta^{\prime a} \eta^{\prime b}(t)$. Then, $M$ is future null geodesically incomplete.

Remarks. If $\Sigma$ is a trapped surface and $M$ obeys the null energy condition, then (5.4) is satisfied for sufficiently small $c$ and we recover the Penrose singularity theorem. The case in which (5.4) holds with $c=0$ corresponds to the codimension 2 case of theorem 2 in [27]. As in theorem 5.1, lemma 3.1 and theorems 4.1 and 4.3 may be used to provide alternative energy conditions that yield singularity theorems similar to theorem 5.2. Finally, note that if the Einstein equations (5.2) hold, then the Ricci curvature term Ric $\left(\eta^{\prime}(t), \eta^{\prime}(t)\right)$ can be replaced by $8 \pi T_{a b} \eta^{\prime a} \eta^{\prime b}(t)$.

Proof of theorem 5.2. We shall be brief, as the general structure of the proof is similar to the proof of the Penrose singularity theorem. See [21] for further details and relevant results from causal theory.

The achronal boundary $\partial J^{+}(\Sigma)$ is a $C^{0}$ achronal hypersurface ruled by null geodesics, i.e. for each point $q \in \partial J^{+}(\Sigma) \backslash \Sigma$ there exists a future directed null geodesic segment from a point in $\Sigma$ to $q$ which is entirely contained in $\partial J^{+}(\Sigma)$. Such null geodesics are called the null generators of $\partial J^{+}(\Sigma)$.

If $\partial J^{+}(\Sigma)$ were compact, then flowing along the integral curves of a timelike vector field on $M$ would establish a homeomorphism between $\partial J^{+}(\Sigma)$ and the Cauchy surface $S$, which would contradict the noncompactness of $S$. Thus, we may assume that $\partial J^{+}(\Sigma)$ is noncompact. Since $\partial J^{+}(\Sigma)$ is closed but noncompact we can find a sequence of points $q_{n}$ in $\partial J^{+}(\Sigma)$ whose $h$-distance to $\Sigma$ (where $h$ is a complete background Riemannnian metric on $M$ ) tends to infinity as $n \rightarrow \infty$. Let $\eta_{n}$ be a null geodesic generator of $\partial J^{+}(\Sigma)$ from $p_{n} \in \Sigma$ to $q_{n} ; \eta_{n}$ must meet $\Sigma$ orthogonally, otherwise the achronality of $\partial J^{+}(\Sigma)$ would be violated. By passing to a subsequence if necessary, we may assume without loss of generality that $p_{n} \rightarrow p \in \Sigma$ and that the initial tangents $\eta_{n}^{\prime}(0) \rightarrow \ell_{+}(p)$.

Let $\eta:[0, a) \rightarrow M, a \in(0, \infty]$, be the affinely parameterized future inextendible null geodesic emanating from $p$ with initial tangent $\ell_{+}(p) ; \eta$ is contained in $\partial J^{+}(\Sigma)$ since each $\eta_{n}$ is. Since $\partial J^{+}(\Sigma)$ is achronal there can be no null focal points, or null focal cut points, to $\Sigma$ along $\eta$. It follows [26] that $\eta$ is contained in a smooth null hypersurface $H \subset \partial J^{+}(\Sigma)$ generated by null geodesics emanating from $\Sigma$ near $p$ with initial tangents given by $\ell_{+}$. Let $K$ be a smooth null vector field on $H$ such that $K=\eta^{\prime}$ along $\eta$, and let $\hat{\theta}$ be the null expansion of $H$ with respect to $K$. Along $\eta, \hat{\theta}=\hat{\theta}(t), t \in[0, a)$, obeys Raychaudhuri's equation (for an irrotational null congruence) [21]:

$$
\frac{\mathrm{d} \hat{\theta}}{\mathrm{d} t}=-\operatorname{Ric}\left(\eta^{\prime}, \eta^{\prime}\right)-2 \hat{\sigma}^{2}-\frac{1}{n-2} \hat{\theta}^{2} .
$$


Now one can argue just as in the proof of theorem 5.1. If $\eta$ were future complete, so that $a=\infty$, then lemma 2.3 and (5.1) would imply that (5.5), with $\hat{\theta}(0)=\theta_{+}(p)$, has no solution on $[0, \infty)$. Hence, $\eta$ must be future incomplete.

\section{Applications and discussion}

We begin with two applications to the Einstein equations coupled to a real scalar field. The minimally coupled Einstein-Klein-Gordon system in $n>2$ spacetime dimensions is given by

$$
R_{a b}-\frac{1}{2} R g_{a b}=8 \pi T_{a b}^{\min }, \quad T_{a b}^{\min }=\nabla_{a} \phi \nabla_{b} \phi-\frac{1}{2} g_{a b}\left(\nabla^{c} \phi \nabla_{c} \phi+m^{2} \phi^{2}\right) .
$$

If $\gamma$ is a unit speed timelike geodesic, then

$$
r(t)=\operatorname{Ric}\left(\gamma^{\prime}, \gamma^{\prime}\right)=8 \pi\left(\left(\nabla_{\gamma^{\prime}} \phi\right)^{2}-\frac{m^{2}}{n-2} \phi^{2}\right),
$$

from which we can see easily that (see, e.g., [28] and [21], p 95) the Klein-Gordon field fails to obey the strong energy condition and hence the standard hypotheses of the Hawking singularity theorem. However, if $\phi$ remains bounded along $\gamma$, with $|\phi| \leqslant \phi_{*}$, say, then we have

$$
-\frac{c}{2}+\int_{0}^{T} \mathrm{e}^{-2 c t /(n-1)} r(t) \mathrm{d} t \geqslant-\frac{c}{2}-\frac{K^{2}}{2 c}
$$

for all $c, T>0$, where $K=m \phi_{*} \sqrt{8 \pi(n-1) /(n-2)}$. The right-hand side has a maximum for $c=K$, and we may conclude from theorem 5.1 that if $\theta<-K$ everywhere on $S$, then $M$ is future timelike geodesically incomplete.

In fact, the same result follows from theorem 4 of [15] (in the $n=4$ case). However, we may also obtain more general results, which both allow for exponential growth of $\phi$ in the proper time along these geodesics and provide finer detail in the case of exponential decay. For example, if $S$ is a smooth compact spacelike Cauchy surface and the scalar field obeys a bound

$$
|\phi(p)| \leqslant \phi_{*} \mathrm{e}^{a \rho(p) /(n-1)}
$$

for $p \in J^{+}(S)$, where $\rho(p)$ is the Lorentzian distance from $p$ to $S$ and $a \in \mathbb{R}$ is constant, then we obtain a bound

$$
-\frac{c}{2}+\int_{0}^{T} \mathrm{e}^{-2 c t /(n-1)} r(t) \mathrm{d} t \geqslant-\frac{c}{2}-\frac{K^{2}}{2(c-a)}
$$

for any $c>a$ and all $T>0$ along $S$-rays, for which $\rho(\gamma(t))=t$. Optimizing over $c>0$ as before, and using theorem 5.1 (and the third remark following its proof) we then have that $M$ is future timelike geodesically incomplete if

$$
\theta< \begin{cases}-a / 2-K & a \geqslant-K \\ K^{2} /(2 a) & a<-K\end{cases}
$$

on $S$. The case $a=0$ corresponds to the result above, while the other cases indicate the additional power of our technique.

A drawback of these results is the need to invoke the supremum $\phi_{*}$ and (if needed) the constant $a$. In principle, they are implicit in the Cauchy data for the Einstein-Klein-Gordon system on $S$. One can also read this result in the following way (with $a=0$ for simplicity): if $S$ is mean contracting, then either $M$ is future timelike geodesically incomplete or $\phi$ exceeds $m^{-1} \sqrt{(8 \pi)^{-1}(n-2) /(n-1)} \inf _{S}|\theta|$ in magnitude somewhere to the future of $S$. 
We now turn to the nonminimally coupled field of mass $m \geqslant 0$ and coupling $\xi$, with stress-energy tensor

$$
T_{a b}=T_{a b}^{\min }+\xi\left(g_{a b} \square-\nabla_{a} \nabla_{b}+\left(R_{a b}-\frac{1}{2} R g_{a b}\right)\right) \phi^{2}
$$

The presence of the Einstein tensor in this expression complicates the initial value problem for the Einstein equations with this source (see [29] for existence and uniqueness results). In addition, the second derivatives allow violations of the NEC as well as the SEC, which can be exploited to construct nonsingular cosmological solutions [30]. However, for $\xi \in[0,1 / 4]$ (including the conformal coupling $\left.\xi=\frac{1}{4}(n-2) /(n-1)\right)$ the theory obeys the inequality

$$
\int_{\gamma} T_{a b} \gamma^{\prime a} \gamma^{\prime b} f(\lambda)^{2} \mathrm{~d} \lambda \geqslant-2 \xi \int_{\gamma}\left\{f^{\prime}(\lambda)^{2}-\frac{1}{2} \operatorname{Ric}\left(\gamma^{\prime}, \gamma^{\prime}\right) f(\lambda)^{2}-\left(\frac{1}{4}-\xi\right) R \gamma^{\prime 2}\right\} \phi^{2} \mathrm{~d} \lambda
$$

for any affinely parameterized causal geodesic $\gamma$ and smooth compactly supported, real-valued $f$ ([31], theorem II.1, modulo change in conventions). If we assume that the field magnitude remains bounded, $|\phi| \leqslant \phi_{*}$ this entails (for the case of null $\gamma$ ) that

$$
\int_{\gamma} \operatorname{Ric}\left(\gamma^{\prime}, \gamma^{\prime}\right)\left(1-8 \pi \xi \phi^{2}\right) f(\lambda)^{2} \mathrm{~d} \lambda \geqslant-16 \pi \xi \phi_{*}^{2}\left\|f^{\prime}\right\|^{2}
$$

for all $f \in C_{0}^{\infty}(\mathbb{R})$. Provided that $\phi_{*}$ is strictly less than the critical value $(8 \pi \xi)^{-1 / 2}$ we may absorb a factor into $f$, thus obtaining

$$
\begin{gathered}
\int_{\gamma} \operatorname{Ric}\left(\gamma^{\prime}, \gamma^{\prime}\right) f(\lambda)^{2} \mathrm{~d} \lambda \geqslant-16 \pi \xi \phi_{*}^{2} \int_{-\infty}^{\infty}\left(\frac{\mathrm{d}}{\mathrm{d} \lambda} \frac{f(\lambda)}{\sqrt{1-8 \pi \xi \phi^{2}}}\right)^{2} \mathrm{~d} \lambda \\
\geqslant-Q\left(\left\|f^{\prime}\right\|^{2}+\tilde{Q}^{2}\|f\|^{2}\right)=:-\||| f \mid\|
\end{gathered}
$$

for all real-valued $f \in C_{0}^{\infty}(\mathbb{R})$, where

$$
Q=\frac{32 \pi \xi \phi_{*}^{2}}{1-8 \pi \xi \phi_{*}^{2}}, \quad \tilde{Q}=\frac{8 \pi \xi \phi_{*} \phi_{*}^{\prime}}{1-8 \pi \xi \phi_{*}^{2}},
$$

and $\phi_{*}^{\prime}$ is an upper bound on $\left|\phi^{\prime}\right|$. (We have used the simple estimate $\left\|(f g)^{\prime}\right\|^{2} \leqslant$ $2\left(\|f\|^{2}\left\|g^{\prime}\right\|_{\infty}^{2}+\left\|f^{\prime}\right\|^{2}\|g\|_{\infty}^{2}\right)$ for any $f \in C_{0}^{\infty}(\mathbb{R}), g \in C^{\infty}(\mathbb{R})$.) This is a bound of the form (4.1) with $r_{0} \equiv 0$ and $r=\operatorname{Ric}\left(\gamma^{\prime}, \gamma^{\prime}\right)$. To proceed, we need the following lemma.

Lemma 6.1. Let $\tau_{0}>0, s>0$ and choose $K>0$ so that

$$
K^{2} \geqslant \tilde{Q}^{2}+Q^{-1} \operatorname{Ric}\left(\gamma^{\prime}, \gamma^{\prime}\right)
$$

on $\left(-\tau_{0}, 0\right]$. For any $\epsilon>0$, there exists $c>0$ and $h \in C^{\infty}(\mathbb{R})$ with supp $h \subset\left[-\tau_{0}, \infty\right)$, $h(t)=\mathrm{e}^{-c t / s}$ on $[0, \infty)$ and

$$
\frac{c}{2}+\||| h \mid\|+\int_{-\tau_{0}}^{0} h^{2} \operatorname{Ric}\left(\gamma^{\prime}, \gamma^{\prime}\right) \mathrm{d} \lambda \leqslant \tilde{Q} \sqrt{Q s+Q^{2} / 2}+\frac{1}{2} Q K \operatorname{coth} K \tau_{0}+\epsilon .
$$

Proof. We take $c=\tilde{Q} s \sqrt{2 Q /(Q+2 s)}$. Then the left-hand side becomes

$$
\tilde{Q} \sqrt{Q s+Q^{2} / 2}+\int_{-\tau_{0}}^{0}\left(Q h^{\prime 2}+\left[Q \tilde{Q}^{2}+\operatorname{Ric}\left(\gamma^{\prime}, \gamma^{\prime}\right)\right] h^{2}\right) \mathrm{d} \lambda
$$

so it is sufficient to show that

$$
\inf _{h} \int_{-\tau_{0}}^{0}\left(h^{\prime 2}+K^{2} h^{2}\right) \mathrm{d} \lambda \leqslant \frac{K}{2} \operatorname{coth} K \tau_{0},
$$

with the infimum taken over the class of $h$ specified in the hypotheses. Treating this as a variational problem, the Euler-Lagrange equation is $h^{\prime \prime}=K^{2} h$ and applying the boundary 
conditions $h\left(-\tau_{0}\right)=0, h(0)=1$, the solution is $h(\lambda)=\left(\sinh K \tau_{0}\right)^{-1} \sinh K\left(\lambda+\tau_{0}\right)$, giving equality in (6.1). This can be approximated arbitrarily well within the given class of $h$.

Accordingly, using theorem 4.1 in place of lemma 2.3, we obtain an analogue of theorem 5.2 if the hypothesis (5.4) is replaced by the requirement

$$
\theta_{ \pm}(p)<-\tilde{Q} \sqrt{(n-2) Q+Q^{2} / 2}-\frac{1}{2} Q K \operatorname{coth} K \tau_{0},
$$

with $K$ computed as above for the extension of the null geodesic $\eta$ to $\left(-\tau_{0}, 0\right]$, for some $\tau_{0}>0$.

Finally, we briefly discuss the potential for applications involving quantum fields. The QEIs established for many free fields are weakened versions of the weak and dominant energy conditions, while our analogue of the Hawking singularity theorem is based on a weakened strong energy condition. In order to prove a Hawking result from QEI hypotheses, it would be necessary to add conditions on the trace of the stress-energy tensor, much as in the case of the classical scalar field discussed above. In addition, it is necessary to find estimates on the timescales for averaging over which the curved spacetime QEIs are well approximated by Minkowski space results. Nonetheless, there seems a good prospect of obtaining results along these lines, and our results provide a proof of principle for the idea that energy conditions based on local averages can be used to deduce singularity theorems. Turning to the Penrosetype results, a significant problem at present is that no locally averaged energy inequality along individual null geodesics is known, and direct analogues of the results for timelike averaging cannot hold in dimensions higher than 2 [32]. Here, it seems that the best approach is to consider averages with a degree of transverse smearing, whereupon QEIs can be proved [32]. It is worth recalling that even the ANEC condition is problematic for averaging along a complete null geodesic: the real scalar field violates ANEC in general spacetimes (see [33] and references therein) although it holds in Minkowski space [13] and in general spacetimes along complete achronal geodesics with a tubular Minkowskian neighbourhood [34]. Moreover, this problem persists even with some transverse averaging schemes [35].

Finally, it should be noted that a full treatment of singularity theorems in the context of quantized matter would require (at least) a semiclassical analysis that takes backreaction into account in a dynamical fashion, bringing significant technical challenges - see [36, 37] for recent results in simple cosmological models and further references, and [38] for positive results on ANEC with transverse smearing in this context ${ }^{5}$.

\section{Acknowledgments}

We are grateful to the Centre for Analysis and Nonlinear PDEs (Maxwell Institute) and the ICMS in Edinburgh for financial support during the workshop Mathematical Relativity at which this work was initiated. It is a pleasure to thank the workshop organizers and participants for useful discussions, particularly Paul Tod and James Vickers. This work was partially supported by NSF grant DMS-0708048.

\section{References}

[1] Penrose R 1965 Phys. Rev. Lett. 14 57-9

[2] Hawking S W 1966 Proc. R. Soc. A 294 511-21

[3] Hawking S W and Penrose R 1970 Proc. R. Soc. A 314 529-48

[4] Barceló C and Visser M 2002 Int. J. Mod. Phys. D $111553-60$

[5] Senovilla J M M 1998 Gen. Rel. Grav. 30 701-848

5 The examples studied in [35] are not solutions to the semiclassical equations. 
[6] Epstein H, Glaser V and Jaffe A 1965 Nuovo Cimento 36 1016-22

[7] Davies P C W and Fulling S A 1977 Proc. R. Soc. A 356 237-57

[8] Fewster C J 2005 Energy inequalities in quantum field theory 14th Int. Congress on Mathematical Physics ed J C Zambrini (Singapore: World Scientific). An expanded and updated version is available as arXiv:math-ph/0501073

[9] Fewster C J, Ford L H and Roman T A 2010 Phys. Rev. D 81121901

[10] Tipler F J 1978 Phys. Rev. D 17 2521-8

[11] Borde A 1987 Class. Quantum Grav. 4 343-56

[12] Roman T A 1988 Phys. Rev. D 37 546-8

[13] Wald R and Yurtsever U 1991 Phys. Rev. D 44 403-16

[14] Penrose R 1972 Techniques of Differential Topology in Relativity (CMBS-NSF Regional Conference Series in Applied Mathematics, vol 7) (Philadelphia, PA: Society for Industrial and Applied Mathematics)

[15] Borde A 1994 Phys. Rev. D 50 3692-702

[16] Galloway G J 1981 Manuscr. Math. 35 209-19

[17] Ford L H 1978 Proc. R. Soc. Lond. A 364 227-36

[18] Roman T A 2006 Some thoughts on energy conditions and wormholes Proc. 10th Marcel Grossmann Conf. on General Relativity ed S Bergliaffa and M Novello (Singapore: World Scientific) (arXiv:gr-qc/0409090)

[19] Fewster C J and Eveson S P 1998 Phys. Rev. D 58084010

[20] Fewster C J 2000 Class. Quantum Grav. 17 1897-911 (arXiv:gr-qc/9910060)

[21] Hawking S W and Ellis G F R 1973 The Large Scale Structure of Space-Time (Cambridge Monographs on Mathematical Physics 1) (London: Cambridge University Press)

[22] Fewster C J and Smith C J 2008 Ann. Henri Poincaré 9 425-55

[23] Ford L H and Roman T A 1996 Phys. Rev. D 53 5496-507

[24] Paeng S H 2009 Math. Proc. Camb. Phil. Soc. 146 475-87

[25] Galloway G J 1986 Math. Proc. Camb. Phil. Soc. 99 367-75

[26] Kemp P M 1984 Focal and focal-cut points PhD Thesis, University of California, San Diego, CA (Ann Arbor, MI: ProQuest LLC)

[27] Galloway G J and Senovilla J M M 2010 Class. Quantum Grav. 27152002 (arXiv:1005.1249)

[28] Bekenstein J D 1975 Phys. Rev. D 11 2072-5

[29] Hübner P 1995 Class. Quantum Grav. 12 791-808

[30] Bekenstein J D 1974 Ann. Phys. 82 535-47

[31] Fewster C J and Osterbrink L W 2006 Phys. Rev. D 74044021

[32] Fewster C J and Roman T A 2003 Phys. Rev. D 67044003

Fewster C J and Roman T A 2009 Phys. Rev. D 80069903 (erratum)

[33] Urban D and Olum K D 2010 Phys. Rev. D 81024039

[34] Fewster C J, Olum K D and Pfenning M J 2007 Phys. Rev. D 75025007

[35] Urban D and Olum K D 2010 Phys. Rev. D 81124004

[36] Pinamonti N 2010 On the initial conditions and solutions of the semiclassical Einstein equations in a cosmological scenario arXiv:1001.0864

[37] Eltzner B and Gottschalk H 2010 Dynamical backreaction in Robertson-Walker spacetime arXiv:1003.3630

[38] Flanagan É É and Wald R M 1996 Phys. Rev. D 54 6233-83 